\title{
Morphological Evaluation of Rat Incisor Enamel and Dentin Induced by Pregnancy and Lactation using A Scanning Electron Microscope
}

\author{
Murat OZBEK ${ }^{1)}$, Sema DURAL ${ }^{1)}$, Aydan $\mathrm{KANLI}^{1)}$, Murvet $\mathrm{TUNCEL}^{2)}$ and Kaan ORHAN ${ }^{3) *}$ \\ ${ }^{1)}$ Department of Oral Diagnosis and Radiology, School of Dentistry, Hacettepe University Sihhiye, 06100, Ankara, \\ ${ }^{2}$ Department of Anatomy, School of Medicine, Hacettepe University, Sihhiye, 06100, Ankara and \\ ${ }^{3)}$ Department of Oral Diagnosis and Radiology, Faculty of Dentistry, Ankara University Besevler, 06500, Ankara, Turkey
}

(Received 6 October 2008/Accepted 24 April 2009)

\begin{abstract}
This study assessed the effects of pregnancy and lactation on the morphology of the dentine tubules and external enamel surface of rat incisor teeth using a scanning electron microscope (SEM) equipped with an energy dispersive x-ray (EDX) system. Twentyfour female Wistar rats were divided into three groups; group A rats were at the end of pregnancy, group B rats were in the post-lactation period and group $\mathrm{C}$ rats, the control group, were unmated. The outer enamel surface and openings of the dentine tubules at the neck regions of the incisors were observed under the SEM and photographed. Examination of the incisor teeth of pregnant and post-lactation rats revealed scratches on the enamel surface. There were few eroded areas and slight changes and the dentine tubules of the pregnant group were fully or partially occluded on the entire surface of the enamel in the lactating rats. Almost all dentine tubules of the rats in this group were open. During the study, EDX analysis of calcium, phosphate and magnesium was also performed at $20 \mathrm{kV}$ and 0 degree tilt. The results of EDX analyses of magnesium were significantly lower in the pregnant group compared with the lactation and control groups for the dentine in the neck region $(\mathrm{p}<0.05)$. The calcium values increased in the lactation group compared with those of the rats in the other two groups $(\mathrm{p}<0.05)$. These results might indicate that changes during pregnancy and lactation affect the content and morphology of mineralized dental hard tissue.
\end{abstract}

KEY WORDS: dentine, enamel, lactation, pregnancy, SEM.

J. Vet. Med. Sci. 71(10): 1273-1277, 2009

The tooth is a convenient model for studying basic biological phenomena such as cell generation, cell differentiation and interaction, synthesis, secretion and organization and mineralization of extracellular matrices. Rat incisor teeth grow continuously and can exhibit all stages of tooth formation at any time. This characteristic makes them ideal models for enamel formation [4]. In addition, in dentine, dentinal tubules play an important role in transferring stimuli and irritants to the pulp. By way of this route and through diffusion in the liquid filling the tubules, various therapeutic agents applied locally to exposed dentin may also reach the pulp [1]. Zeni et al. [16] examined bone mass changes in the total, axial and appendicular skeleton as well as in the different subareas of the femur and tibia in rats kept on a diet including normal amounts of calcium. Their experimental study using dual energy absorptiometry in vivo to visualize changes in BMD during the reproductive cycle of the rat showed that normal pregnancy in the rat appears to have little influence on bone, whereas lactation induces significant bone loss, mainly in areas with predominant trabecular bone [16]. Moreover, Miller et al. [5] demonstrated that significant changes occur in the rates of dentin apposition in the continuously erupting incisors of rats during pregnancy and lactation. The rate of dentin apposition in the rat is known to be sensitive to endocrinal alterations [13], and it would therefore seem to provide a good model

* Correspondence to: Dr. Orhan, K., University of Ankara, Faculty of Dentistry, Department of Oral Diagnosis and Radiology, 06500 Besevler, Ankara, Turkey.

e-mail : cal153@yahoo.com for determining whether pregnancy and lactation in rats alter the rates of accretion of maternal mineralizing tissue [5]. Information about the effects of lactation and pregnancy on the morphologies of dentin and enamel in the rat is very limited in the literature.

In this study, we examined, by scanning electron microscope (SEM), the morphological changes in maternal rat incisor dentine and enamel during pregnancy and at the end of the lactation period and performed an energy dispersive $\mathrm{x}$-ray (EDX) analysis to evaluate calcium, phosphate and magnesium levels of the surfaces of the dentine and enamel.

\section{MATERIALS AND METHODS}

Experiments involving live animals were carried out with the approval of the Institutional Animal Care and Use Committee (2001/70-3) and according to Hacettepe University Animal Experimentation Regulations. A total of $24(n=24)$ female Wistar rats aged 12-14 weeks (each weighing approximately 200-250 g) were used in accordance with the guidelines for the care and use of experimental animals at the Medical Faculty of Hacettepe University. The rats were supplied by the Experimental Animals Section of the Medical Faculty of Hacettepe University. Wistar rats reach puberty in 12 weeks, have a gestation period 20-22 days, a lactation period of approximately 28 days, and have a life span of 2-3.5 years [9]. Their incisors grow approximately $631 \mu \mathrm{m}$ per day [12].

The animals were maintained at a certain temperature (23 $\pm 1^{\circ} \mathrm{C}$ ), with $55 \%$ humidity and a light/dark cycle of $12 \mathrm{hr}$ 
(automatically controlled). They were fed a purified rat diet containing $1.1 \%$ calcium and $0.8 \%$ phosphate (Kalecik Yem $^{\circledR}$, Turkey) and water ad libitum. They were divided into the following three groups: the animals in Group A $(n=8)$ were at the end of pregnancy $(90+20$ days $)$, the animals in Group B $(n=8)$ were in the post-lactation period $(90+20+28$ days $)$ and the animals in Group $C(n=8)$ were unmated rats (the control group, $90+20$ days). The pregnancies of the rats were confirmed by the presence of sperm in vaginal smears, observation by a veterinary technician and evaluation with ultrasound. On the 20th day, the rats in Groups $\mathrm{A}$ and $\mathrm{C}$ were sacrificed by administration of an anesthetic solution (Ketalar, $50 \mathrm{mg} / \mathrm{ml}$, Parke-Davis, Istanbul, Turkey, and Rompun 2\%, Bayer, Istanbul, Turkey). Group B rats were anesthetized and sacrificed by administration of the same anesthetic solution at the 28th day of lactation. All procedures were performed in the morning to minimize the effects of circadian variations. The right mandibular incisor teeth of the rats were then extracted. The extracted incisors were fractured in an oblique direction using forceps. The teeth were not sectioned in order to protect the incisors from the smoothing effect of the blade edge as well as to prevent formation of a smear layer. Half of each tooth was mounted on a specimen stub with the external labial (vestibular) surface of the enamel directed upwards for surface examination. The other half of each tooth was mounted on stubs with the internal surfaces facing up so that the dentine tubules could be observed. All specimens were fixed on stubs using a double-sided band, and conductive silver was applied all around them. They were then sputtered with $150 \mathrm{~nm}$ gold-palladium, kept overnight in a high vacuum environment and examined using a Jeol JSM 6400 (Tokyo, Japan; SEM). The outer enamel surface and openings of the dentine tubules at the neck regions of the incisors were observed under the SEM and photographed. During the observation, EDX analysis of calcium, phosphate and magnesium was also performed at $20 \mathrm{kV}$ and 0 degree tilt using the Jeol JSM 6400 scanning electron microscope. EDX analysis was performed for both the enamel and dentin levels in the neck region. The results of the EDX analyses were evaluated by one-way Anova test. The data obtained for the calcium, phosphate and magnesium elements of the dentine were analyzed by Tukey's test.

\section{RESULTS}

There were no statistical differences in the incisor neck region enamel tissue with regards to calcium ( $\mathrm{p}=0.082$, $\mathrm{p}>0.05)$ and phosphate $(\mathrm{p}=0.373, \mathrm{p}>0.05)$ between the control, pregnant and lactation groups. EDX studies of the dentine in the neck region $(62.53 \pm 2.265,65.17 \pm 3.031$ and $70.54 \pm 1.362$ for calcium in the control, pregnancy and lactation groups, respectively; $35.75 \pm 2.132,33.935 \pm 2.081$ and $27.21 \pm 0.923$ for phosphate in the control, pregnancy and lactation groups, respectively; and $1.72 \pm 0.201,0.895 \pm$ 0.1342 and $2.25 \pm 0.594$ for magnesium in the control, pregnancy and lactation groups, respectively) showed a statisti- cally significant difference $(\mathrm{p}<0.05)$. Compared with the control and lactation groups, the calcium values were increased and the phosphate values were decreased in the lactation group. Compared with the control group, the magnesium values were significantly lower in the pregnancy group and higher in the lactation group ( $\mathrm{p}<0.05$; Fig. 1).

External labial surfaces of the rat incisor teeth were studied with by scanning electron microscopy, and representative samples of each study group were photographed and presented in this paper. Almost the entire outer surfaces of the enamel on the neck region (the neck region was the apical area closest as possible to the apex of the teeth in order to assure exposure of the area's most influenced by the stresses of lactation and pregnancy) of the incisors were smooth in the control group. There were some small, nonspecific artifacts around the enamel surface and a few scratches and contusions in some areas (Fig. 2). Examinations under larger magnifications were performed to con-
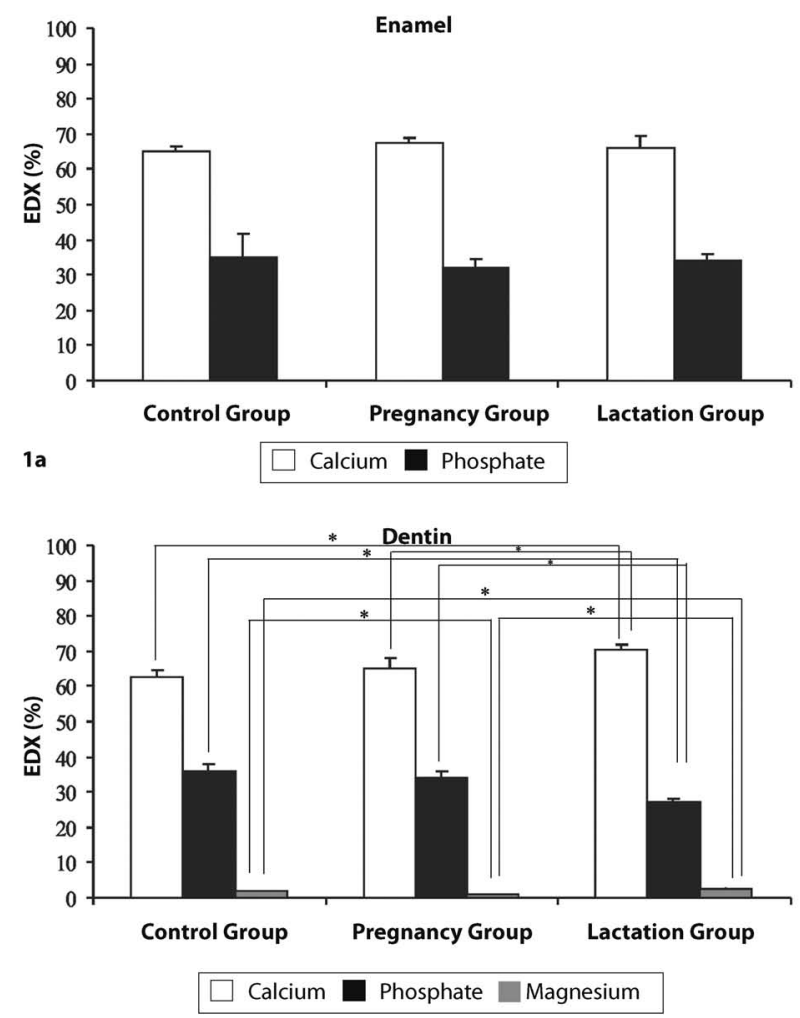

(*) Tukey test results: ( $s=$ significant, $n s=$ non-significant) (Control group $=\mathrm{C}$, Pregnancy group $=\mathrm{P}$, Lactation group $=\mathrm{L}$ )

$\begin{array}{llll} & \text { Calcium: } & \text { Phosphate: } & \text { Magnesium: } \\ C-P(n s) & C-P(n s) & C-P(s) \\ C-L(s) & C-L(s) & C-L(s) \\ \text { b } & P-L(s) & P-L(s) & P-L(s)\end{array}$

Fig. 1. Results of the EDX analysis for enamel. There were no significant differences with regards to the calcium $(p=0.082$, $p>0.05)$ and phosphate $(p=0.373, p>0.05)$ elements between the control, pregnant and lactation groups (Fig. 1a); for dentine, significantly higher level calcium and magnesium values were found in the lactation group $\left({ }^{*} \mathrm{p}<0.05\right.$; Fig. 1b). 


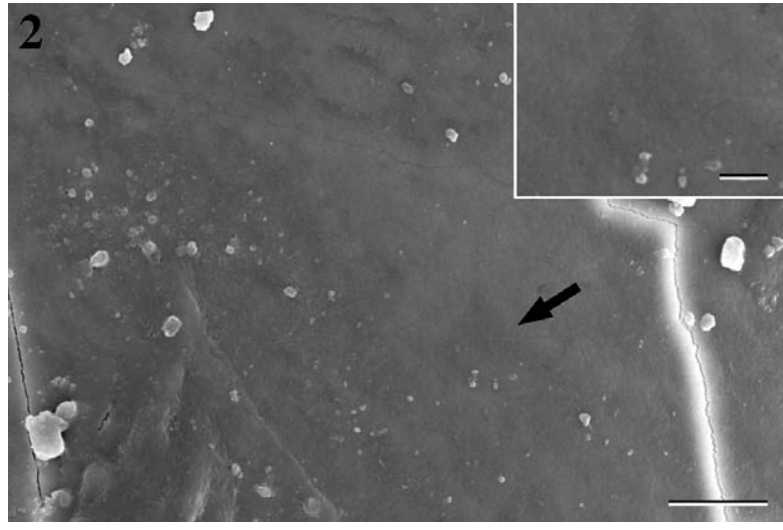

Fig. 2. Smooth outer surface of the enamel on the neck region of an incisor of a control rat. Bar $=10 \mu \mathrm{m}$. The inset also shows the smooth surface of the incisor (arrow).

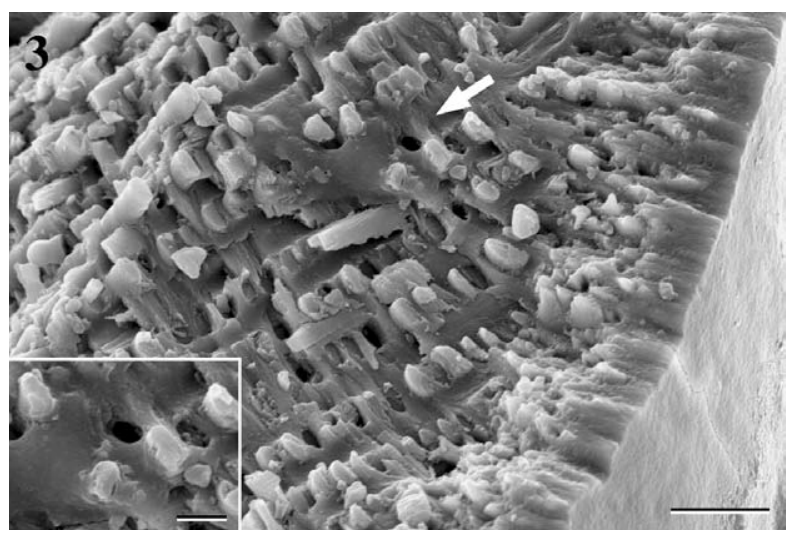

Fig. 3. Cross sections of dentin tubules on the oblique fractured face of the dentin layer of rat incisor teeth in the control group. The tubules are open. Bar $=10 \mu \mathrm{m}$. The inset shows open dentin tubules (arrow).

firm the incisors smooth surface. Cross sections of the dentin tubules were also observed on the oblique fractured face of the dentin layer in the control rats. These tubules were open in the unmated rats (Fig. 3). SEM observations of the pregnant group showed that there were many changes, such as scratches and contusions on the enamel surface. These changes were found on the entire enamel surface, with no smooth areas. Severely eroded and destructed areas were also seen in some parts of the enamel surface. Higher magnifications also revealed scratches on the enamel surface (Fig. 4). The dentine tubules of the incisor teeth of the pregnant rats were mostly occluded. The cross-sectional appearance of the fractured dentin surface demonstrated that almost all dentine tubules were fully or partially occluded (Fig. 5). Similar observations were also performed for the rats in the post-lactation period. In contrast with the pregnant rats, there were few eroded areas in these specimens, and the changes were slight on the entire surface of the enamel (Fig. 6). Almost all dentine tubules in this animal

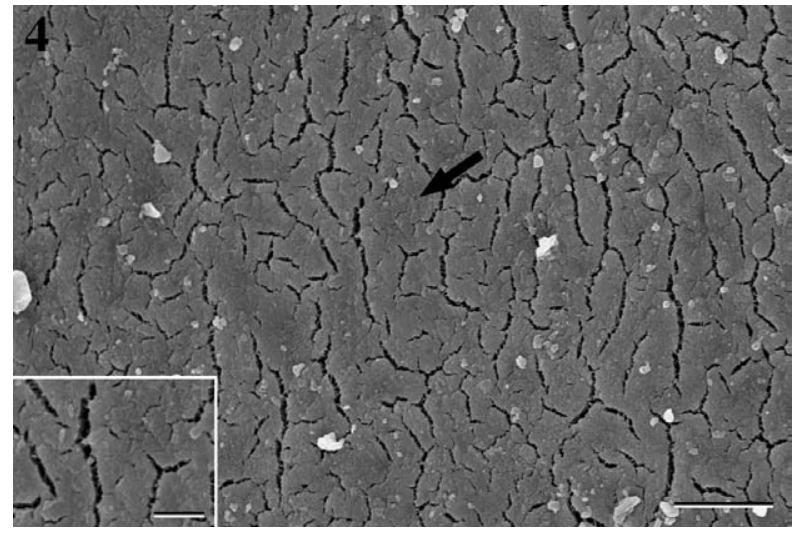

Fig. 4. Degenerative changes such as scratches and cracks on the enamel surface of the pregnant rat. Bar $=10 \mu \mathrm{m}$. The inset shows scratches and cracks (arrow). Severely eroded and destructed areas of the enamel surface of the pregnant rat.

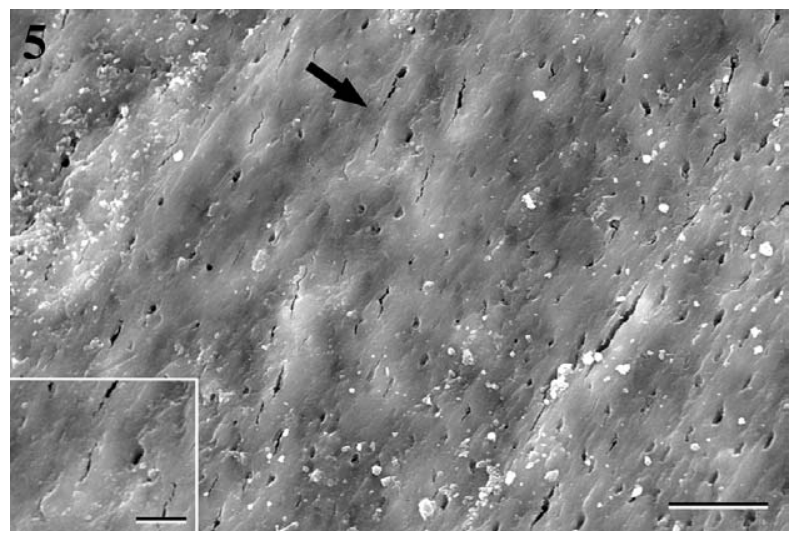

Fig. 5. Occluded dentin tubules of pregnant rat incisor teeth. Bar $=10 \mu \mathrm{m}$. The isent shows an occluded dentin tubule (arrow).

group were open, although a few dentine tubules were partially occluded (Fig. 7).

\section{DISCUSSION}

Calcium is an essential nutrient that is involved in most metabolic processes and together with phosphate salts provides mechanical rigidity to the bones and teeth, where $99 \%$ of the body's calcium is stored. The calcium in the skeleton carries the additional role of acting as a reserve supply of calcium to meet the body's metabolic needs when there is a lack of calcium [6]. There are significant alterations in calcium metabolism during pregnancy and lactation in mammals. Calcium absorption is increased to compensate for calcium loss through placental transfer and breast-feeding. It is not clear whether the deficiency of calcium during pregnancy and postpartal lactation is linked to postmenopausal osteoporosis. The mechanism of post-pregnancy osteoporosis is not clear and needs to be studied in terms of both prevention and treatment $[11,15]$. In a previous study we 


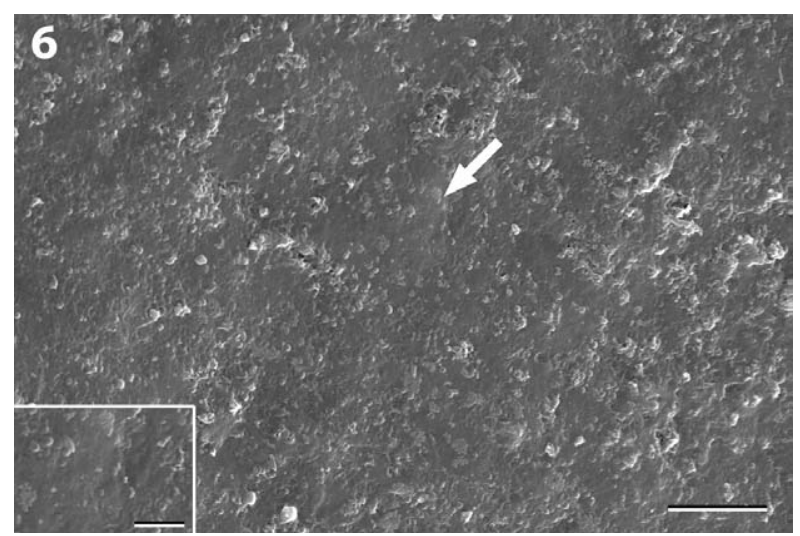

Fig. 6. Eroded areas and slight degenerative changes on the surface of the enamel of the rat during lactation. Bar $=10 \mu \mathrm{m}$. The inset shows slight erosion on the surface (arrow).

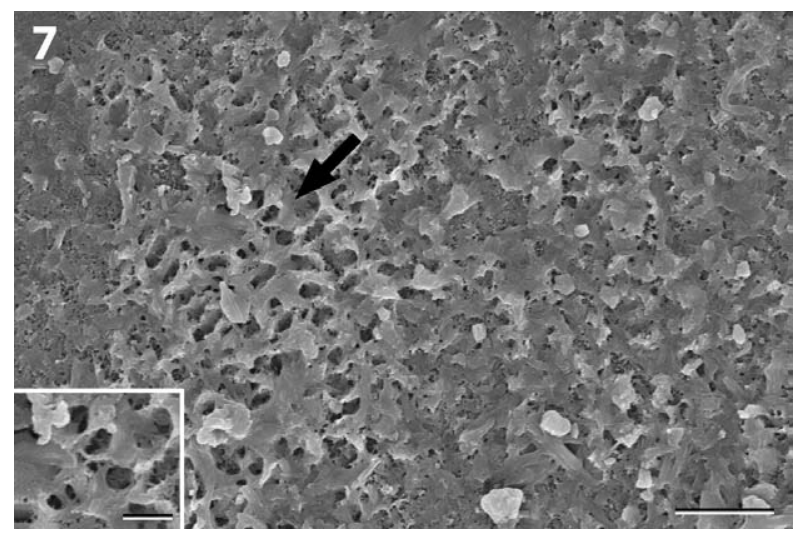

Fig. 7. Partially occluded dentine tubules on the oblique fractured face of the dentin layer of rat incisor teeth in the lactation period. $B a r=10 \mu \mathrm{m}$. The inset shows partially occluded dentin tubules (arrow).

conducted on entire specimens of rat molar teeth to examine both the enamel and the dentin, we found that there were significant increases in the calcium and magnesium contents in the pregnant group, while the zinc content of the rats in this group showed no significant difference compared with the control group [8]. In our study, the results of EDX analyses revealed no significant differences in the incisor neck region enamel with regards to the calcium and phosphate elements of the control, pregnant and lactation groups. However, in the EDX studies carried out on the dentine in the neck region, a statistically meaningful difference was observed. In comparison the control and lactation group teeth, it was seen that the calcium values increased in the lactation group, while a decrease was observed in the phosphate values. Comparing again the pregnant and lactation group teeth in particular, the calcium values were higher in the lactation group compared with pregnant group, and the phosphate values were lower. When we compared the control and pregnant groups, we found that the pregnant group had lower levels of magnesium, which may be attributed to the fetus accumulating minerals. The prism pattern in the inner enamel of adult rat incisors was studied by Risnes using SEM; reported occasionally observation of crystallite bridges connecting adjacent prisms [10]. The prisms are arranged in single-layered rows that are oriented transversely along the long axis of the tooth. In the outer part of the enamel, all prisms are parallel to each other and run in an incisal direction at an angle of about $20-25^{0}$ relative to the dentino-enamel junction. The outer $5 \mu \mathrm{m}$ of the enamel is devoid of prisms [14]. The pre dentin area displays a spongy structure that contrasts with the compact appearance of the dentin [3]. In the rat incisors, the number of tubules in the incisal dentin was slightly less than that in the dentin of the middle part of the crown. The tubules in the dentin of rat incisors exhibited local irregularities and ramifications. The mean diameter of the dentin tubules near the pulpal wall in the rat incisors was $1.7 \mu \mathrm{m}$, and the number of tubules/ $\mathrm{mm}^{2}$ was 51 [1]. The volume of incisor dentin formation was semi-longitudinally studied by Futatsuki et al. [2] using the time-marking method during the reproductive cycle, including pregnancy, parturition, lactation and post-weaning, in mice and was compared with unmated, non-pregnant control mice. In the control animals, there were no significant differences in the periods throughout the experiment. However, the volume of dentin formation increased during the reproductive cycle more than it did in the control and the increase in volume was significant. In addition, during pregnancy, the volume increased gradually, with the greatest increase seen in the last 4 days of pregnancy, including the day of parturition, as compared with the first half-term of pregnancy. The volume decreased immediately after parturition compared with the second half-term of pregnancy. It was suggested that the volume of dentin formation is affected by the change in maternal metabolism during the reproductive cycle in mice. Miller et al. [5] stated that the rate of dentin apposition is significantly increased in pregnant rats compared with age-matched, non-matched controls during the first week of pregnancy and appeared to reach a peak during the second week. They suggested that irrespective of the endocrine basis of the changes observed in their study, it was apparent that pregnancy and lactation affect the rate of maternal dentin apposition in the rat.

As a result of this increase in dentine apposition, as previously detected by these other researchers during the pregnancy period, the microscopic observations of our study showed that the dentine tubules of the neck region of the pregnant rats were mostly occluded. The cross-sectional appearance of the fractured dentin surface demonstrated that almost all dentine tubules were either partially or fully occluded.

Pregnancy and lactation affect the rate of maternal dentine apposition and mineralization in the rat. Incisal sections of the incisors revealed that the pregnant and lactation groups' enamel mineralization values were increased compared with those of the control group, while the pregnancy and post-lactation period dentine values were decreased slightly compared with the control group. In the neck sec- 
tions, the pregnant and lactation groups' enamel values were lower compared with those of the control group; pregnancy and post-lactation period dentine values also decreased slightly compared with the control group. During the postlactation period, the neck section dentine mineralization was decreased compared with the incisal section. In sum, the enamel and dentine layers of rat incisors were affected to varying degrees by these changes in mineralization levels during the maternal period [7]. Turnbull et al. [13] stated that few hormones are known to stimulate dentin apposition directly. We are convinced that hard tissue calcium accumulation has a role in the morphological alterations we have detected in rat teeth during the pregnancy and lactation periods.

In conclusion, the results of this study revealed many degenerative changes, including severely eroded and destructed areas on the enamel surface in pregnant rats. Dentine tubules were mostly occluded. In comparison with the pregnant rats, the enamel surfaces of the post-lactation group rats were smoother, with wider dentine tubules. The calcium and phosphate levels of the surfaces of the neck regions of enamel did not differ in terms of the EDX analysis results, but in the dentine in the neck region, the existence of a statistically significant difference was observed between the control group and the pregnancy and lactation groups. It is our belief that the differences observed in the dentine may be due to changes in the tubular structure, induced by the alterations in calcium metabolism observed during the pregnancy or lactation period.

\section{REFERENCES}

1. Forssell-Ahlberg, K., Brännström, M. and Edwall, L. 1975. The diameter and number of dentinal tubules in rat, cat, dog and monkey. Acta. Odontol. Scand. 33: 243-250.

2. Futatsuki, T., Matsumoto, T. and Nakata, M. 1995. Change of dentine formation during the reproductive cycle in mice. $J$. Craniofac. Genet. Dev. Biol. 15: 51-56.

3. Hansson, H.A., Linde, A. and Nygren, H. 1975. Scanning elec- tron microscopic studies of the rat incisor odontoblastema. Cell Tissue Res. 159: 233-243.

4. Leblond, C.P. and Warshawsky, H. 1979. Dynamics of enamel formation in the rat incisor tooth. J. Dent. Res. 58: 950-975.

5. Miller, S.C., Omura, T.H. and Smith, L.J. 1985. Changes in dentin appositional rates during pregnancy and lactation in rats. J. Dent. Res. 64: 1062-1064.

6. Nordin, B.E. 1997. Calcium and Osteoporosis. Nutrition 13: 664-686.

7. Ozbek, M., Kanli, A., Dural, S., Sahin, I., Gonen, E. and Tulunoglu, I. 2004. Effects of pregnancy and lactation on the microhardness of rat incisor dentine and enamel. Arch. Oral Biol. 49: 607-612.

8. Ozbek, M., Sahin, I., Kanli, A., Dural, S. and Serter, R. 2001. Changes in calcium, magnesium and zinc content on rat molar teeth during pregnancy. Hacettepe Univ. Dental J. 25: 3-7.

9. Pass, D. and Freeth, G. 1993. The Rat. Anzccart News 6: 1-4.

10. Risnes, S. 1979. A scanning electron microscope study of aberrations in the prism pattern of rat incisor inner enamel. Am. J. Ant. 154: 419-436.

11. Smith, R., Stevenson, J.C., Winearls, C.G., Woods, C.G. and Wordsworth, B.P. 1985 Osteoporosis of pregnancy. Lancet 1: $1178-1180$.

12. Smith, C.E. and Warshawsky, H. 1975. Cellular renewal in the enamel organ and the odontoblast layer in the rat incisor as followed by radioautography using $\mathrm{H} 3$ thymidine. Anat. Rec. 183: 523-561.

13. Turnbull, R.S., Heersche, J.N., Tam, C.S. and Howley, T.P. 1983. Parathyroid hormone stimulates dentin and bone apposition in the thyroparathyroidectomized rat in a dose-dependent fashion. Calcif. Tissue Int. 35: 586-590.

14. Warshawsky, H. and Smith, C.E. 1971. A three-dimensional reconstruction of the rods in rat maxillary incisor enamel. Anat. Rec. 169: 585-592.

15. Yamamoto, N., Takahashi, H.E., Tanizawa, T., Kawashima, T. and Endo, N. 1994 Bone mineral density and bone histomorphometric assessments of postpregnancy osteoporosis: a report of five patients. Calcif. Tissue Int. 54: 20-25.

16. Zeni, S.N., Di Gregorio, S. and Mautalen, C. 1999. Bone mass changes during pregnancy and lactation in the rat. Bone $\mathbf{2 5}$ : $681-685$. 